Bulletin of the Section of Logic

Volume 49/4 (2020), pp. 327-342

http://dx.doi.org/10.18778/0138-0680.2020.23

David Makinson

\title{
THE PHENOMENOLOGY \\ OF SECOND-LEVEL INFERENCE: \\ PERFUMES IN THE DEDUCTIVE GARDEN
}

\begin{abstract}
We comment on certain features that second-level inference rules commonly used in mathematical proof sometimes have, sometimes lack: suppositions, indirectness, goal-simplification, goal-preservation and premise-preservation. The emphasis is on the roles of these features, which we call 'perfumes', in mathematical practice rather than on the space of all formal possibilities, deployment in prooftheory, or conventions for display in systems of natural deduction.

Keywords: Second-level inference, suppositions, indirect inference, goal simplification, goal preservation, wlog, premise preservation.

\section{Introduction}

In logic, it is commonplace to distinguish between inferences of first and second levels. In broad terms, a first-level inference passes from certain statements serving as premises to a statement taken as conclusion. In concise notation, it is of the type $\Gamma \vdash \gamma$, where $\gamma$ is a statement and $\Gamma$ is a finite set of the same, with the sign $\vdash$ indicating passage from one to the other. A second-level inference, on the other hand infers the validity of an entire argument from the validity of one or more other arguments. In the same notation, it is of the form $\Gamma_{i} \vdash \gamma_{i}(i \leq n) / \Delta \vdash \delta$ where the slash marks a step from the $n$ subordinate inferences $\Gamma_{i} \vdash \gamma_{i}$ on the left to the principal one on the right. The former kind of inference can, of course, be regarded
\end{abstract}

Presented by: Andrzej Indrzejczak

Received: July 15, 2020

Published online: November 10, 2020

(C) Copyright for this edition by Uniwersytet Łódzki, Łódź 2020 
as the limiting case of the latter where $n=0$, but here we will always be considering the principle case that $n \geq 1$.

Not only are these two levels formally distinct, but they also 'smell' quite differently. One also senses differences within the second level; for example, each of the rules of conditional proof $\left(C P, \rightarrow^{+}\right)$, reductio ad absurdum $(R A A)$, disjunctive proof $\left(D P, \vee^{+}\right)$, universal generalization $\left(U G, \forall^{+}\right)$and existential instantiation $\left(E I, \exists^{-}\right)$has its own intuitive feel or, as we shall say, its 'perfume'. This article is about such perfumes, which can be defined quite precisely. We will see how a second-level inference-rule may be (or fail to be) suppositional, indirect, goal-simplifying, goal-preserving or premise-preserving, in senses to be defined, discuss why these features can be useful in practice, and draw attention to a connection with without loss of generality (wlog) reasoning.

Some disclaimers may forestall misunderstanding. There are no theorems, no philosophical messages or agenda. We are not interested in the space of all mathematically possible forms of second-level proof, but with steps that, arguably, one finds in everyday mathematical reasoning. Since that proceeds in accord with classical logic, it is the only kind of logic to concern us, not free, intuitionistic, relevance-sensitive, paraconsistent, fuzzy or any other variety. Nor are we concerned with the various display systems that have been devised in textbook presentations of natural deduction (see the overviews in e.g. [10], [3, chapter 2], [11]). The text merely offers an organized review of folklore about inferential practice; in the more pretentious language of our title, it is a foray in the phenomenology of second-level inference.

As is well known, any second-level inference rule $\Gamma_{i} \vdash \gamma_{i}(i \leq n) / \Delta \vdash \delta$ may equivalently be presented as what has been called a 'split-level' rule $\Delta ; \Gamma_{i} \vdash \gamma_{i}(i \leq n) / \delta$, where the premise-set $\Delta$ of the principal inference is moved to the left of the slash (cf. [8, chapter 10]). In the split-level version the conclusion is thus a proposition $\delta$ (as in a first-level rule), which is obtained from a set $\Delta$ of propositions (again like the first level) that is, however, accompanied by the inferences $\Gamma_{i} \vdash \gamma_{i}$ (as for the second level). Arguably, this is the form that corresponds most closely to inferential practice and to what is done in systems of natural deduction. The present text could be written using either idiom; we have chosen the second-level one because it is a little easier to read and more familiar to readers.

Throughout, we use the terms 'proof', 'inference' and 'argument' interchangeably according to the whims of style; similarly with 'subordinate 
inference' and 'sub-proof', 'statement' and 'proposition' (understood in a broad sense, as possibly containing free variables), 'supposition', 'assumption', and 'hypothesis', while recognizing that in other contexts it can be useful, even essential, to make distinctions between them. Table 1, at the end of the paper, keeps track of the discussion.

\section{Suppositions}

Do second-level proofs have anything in common apart from the general type $\Gamma_{i} \vdash \gamma_{i}(i \leq n) / \Delta \vdash \delta$ mentioned above? It is tempting to say that, in every instance, each of the subordinate inferences $\Gamma_{i} \vdash \gamma_{i}$ makes a supposition (also called assumption or hypothesis); in other words, that for all $i \leq n$, there is a statement $\alpha$ with $\alpha \in \Gamma_{i}$ but $\alpha \notin \Delta$.

That is almost true but, notoriously, not quite. There is a very important form of second-level inference, with just one subordinate inference, that makes no supposition, namely universal generalization $\left(U G, \forall^{+}\right)$:

$$
U G: \Gamma \vdash \gamma / \Gamma \vdash \forall x(\gamma) \text {, when } x \text { has no free occurrences in } \Gamma \text {. }
$$

Here the premise-set $\Gamma$ of the unique subordinate inference $\Gamma \vdash \gamma$ is exactly the same as that of the principal inference $\Gamma \vdash \forall x(\gamma)$, nothing more, nothing less.

From a heuristic point of view, the presence of a supposition in a subordinate argument gives it one more item to grab and deploy, so the absence of a supposition means that it foregoes that bonus. But $U G$ has another feature that tends to compensate: it is 'goal-simplifying', in the sense that the conclusion $\gamma$ of its subordinate argument is simpler, in its logical structure, than the conclusion $\forall x(\gamma)$ of the principal argument. We will return to goal-simplification in section 4 .

All other rules for logical connectives in Table 1 are suppositional, as can be seen by inspection. For disjunctive proof,

$$
D P, \vee^{-}: \Gamma \cup\{\alpha\} \vdash \gamma ; \Gamma \cup\{\beta\} \vdash \gamma / \Gamma \cup\{\alpha \vee \beta\} \vdash \gamma,
$$

the suppositions are $\alpha, \beta$. Another form of disjunctive proof widely used in informal practice, sometimes called proof by cases, is $D P x: \Gamma, \alpha \vdash \gamma$; $\Gamma, \neg \alpha \vdash \gamma / \Gamma \vdash \gamma$, where the suppositions are $\alpha, \neg \alpha$. For existential instantiation, the supposition is $\alpha$ : 


$$
\begin{aligned}
& E I, \exists^{-}: \Gamma \cup\{\alpha\} \vdash \gamma / \Gamma \cup\{\exists x(\alpha)\} \vdash \gamma, \text { when } x \text { has no free } \\
& \text { occurrences in } \Gamma, \gamma .
\end{aligned}
$$

For the two forms of conditional proof in Table 1, the suppositions are respectively $\alpha, \neg \gamma$ :

$$
\begin{gathered}
C P a, \rightarrow^{+}: \Gamma \cup\{\alpha\} \vdash \gamma / \Gamma \vdash \alpha \rightarrow \gamma \\
C P b, \rightarrow^{+}: \Gamma \cup\{\neg \gamma\} \vdash \neg \alpha / \Gamma \vdash \alpha \rightarrow \gamma .
\end{gathered}
$$

For reductio ad absurdum, the supposition is $\neg \alpha$ in each of the two subordinate arguments of $R A A a$, as also in the unique subordinate argument of $R A A b$ :

$$
\begin{gathered}
R A A a: \Gamma \cup\{\neg \alpha\} \vdash \gamma ; \Gamma \cup\{\neg \alpha\} \vdash \neg \gamma / \Gamma \vdash \alpha \\
R A A b: \Gamma \cup\{\neg \alpha\} \vdash \alpha / \Gamma \vdash \alpha .
\end{gathered}
$$

We have distinguished between two forms of $C P$, and two forms of $R A A$, because they disagree on other perfumes to be considered later. On the other hand, we do not distinguish between the above forms of $R A A$ and those obtained by inverting the positive and negative occurrences of $\alpha$. Although the latter distinction is important for intuitionistic logic, we remain in the classical domain and it turns out that the inverted forms agree with the above ones on all perfumes considered.

What about connective-free (sometimes called structural) rules? Reflexivity is connective-free, but first-degree, while both monotony and cumulative transitivity are second-degree:

$$
\begin{aligned}
& \text { Monotony }: \Gamma \vdash \gamma / \Gamma \cup \Delta \vdash \gamma, \\
& C T: \Gamma \vdash \gamma ; \Gamma \cup\{\gamma\} \vdash \delta / \Gamma \vdash \delta .
\end{aligned}
$$

Their status is rather special, in inferential practice as much as in logical theory. They are less visible than the rules for connectives in everyday mathematical reasoning, as well as in systems of natural deduction, because they are rarely rendered explicit when applied. In effect, they are implicit in the very structure of a deduction as it develops, whether in the usual linear fashion or in tree form. Moreover, in the present author's view, their connections with the various perfumes that we are considering are less interesting than in the case of rules for connectives. For these reasons, we settle on a compromise: the behaviour of these two rules with respect 
to each perfume is recorded in Table 1 (last two rows), but not discussed further in the text.

The following remarks may help put this bare picture in perspective.

History. It is interesting to recall that Jaśkowski, in his seminal paper of 1934 ([5]), appears to have been reluctant to accept that there are secondlevel inferences without suppositions. The title of his paper is "On the rules of suppositions in formal logic". When presenting $U G$, he introduces its subordinate inference by writing $T x$ on a new line, explaining that $T$ "is here a new constant analogous to the symbol of supposition $S$ " (section 5, page 29).

To be sure, choosing an item arbitrarily by declaring a fresh variable does have some resemblance to the act of making a supposition. This is reflected in the language used: in English, at least, both "let $x$ be..." and "suppose that..." are in the imperative rather than the indicative mood. But they are not quite the same action, and a 'semi-supposition' display risks obscuring the difference.

In effect, we can read Jaśkowski's $T x$ entry in either of two ways. On one reading, which is rather confusing, it articulates the constraint on the variable $x$, which is a condition in the metalanguage, as if it were an additional premise of the subordinate inference. On another reading, it merely announces that one is about to enter a subordinate argument and that one intends to generalize on the variable $x$ when leaving it - which is what is also done by the informal mathematical phrase "let $x$ be an arbitrary so-and-so". However, giving $T x$ a line number in a derivation may not be the most transparent way of signalling that reading.

In any case, the $T x$ notation was not used in the independently conceived work of Gentzen [1], which was much more influential for theoretical investigations of mathematical logicians. And although Jaśkowski's paper was, directly or indirectly, a basic inspiration for textbook accounts of natural deduction for students of philosophy in the second half of the twentieth century, few of them adopted this part of his notation, one well-known exception being, however, [6] (cf. [4]).

Flattening. On the other hand, textbooks presenting systems of natural deduction often streamline displays by "flattening" $U G$, that is, transforming it into a step that is first-level, but procedural rather than inferential. The rule is articulated as authorizing passage from a proposition $\gamma$ to the corresponding proposition $\forall x(\gamma)$ under the proviso that $x$ does not 
occur free in any of the premises or suppositions on which $\gamma$ depends in the deduction under construction. That the passage is procedural rather than inferential is evident from the fact that in general $\gamma \not \forall \forall x(\gamma)$, irrespective of whether the proviso is satisfied. It is only when we reflect on the rationale for the proviso that we can begin to see the second-level inference underlying the procedural step.

While such flattening simplifies the formal display of a natural deduction, in the present author's view it runs a danger of obscuring what is really going on. Given that we have deduced $\gamma$ from various premises and suppositions (the $\Gamma$ in the rule) in which $x$ does not occur free, $U G$ authorizes us to conclude $\forall x(\gamma)$ on the basis of those same propositions. That is second-level and the student should brought to realize it, and not allowed to forget it. In particular, $U G$ should not be confused with the first-level and genuinely inferential step of vacuous generalization $V G: \gamma / \forall x(\gamma)$ whenever $x$ has no free occurrences in $\gamma$.

Universal generalization is not the only second-level rule that systems of natural deduction like to flatten. Even more so is existential instantiation which, we recall, tells us: $\Gamma \cup\{\alpha\} \vdash \gamma / \Gamma \cup\{\exists x(\alpha)\} \vdash \gamma$, when $x$ has no free occurrences in $\Gamma, \gamma$. This is treated as authorizing passage (again, procedurally, but not inferentially) from a proposition $\exists x(\alpha)$ to the corresponding proposition $\alpha$, under a suitable condition. The precise formulation of that condition varies with the conventions of the particular natural deduction system, but its essential content is that $x$ does not occur free in any of the premises or suppositions on which $\exists x(\alpha)$ depends, nor on any conclusion that is subsequently derived from $\alpha$. This reference to premises and conclusions again alerts us to the fact that that there is a second-level inference underlying the first-level procedural step.

Colloquial mathematical reasoning with $E I$ also flattens it, in a less formal way. In a proof, having reached a proposition $\exists x(\alpha)$, where $\alpha$ contains $x$ free, one simply says "choose any one such $x$ " and works on $\alpha$, taking care not to use the same variable $x$ for anything else until the proof is complete. The second-level nature of the manoeuvre is thus left implicit, and perhaps corresponds more closely to a variant of $E I$, namely the rule $\Gamma \vdash \exists x(\alpha), \Gamma \cup\{\alpha\} \vdash \gamma / \Gamma \vdash \gamma$, under the same proviso that $x$ has no free occurrences in $\Gamma, \gamma$. There is more on 'flattening' second-level and split-level rules in [8, section 10.3.3]. 
Making UG suppositional. It is also possible to reformulate $U G$ to render it suppositional. We may add to the premise-set $\Gamma$ of the subordinate inference $\Gamma \vdash \gamma$, as a supposition, either $\neg \gamma$, or a tautology of classical propositional logic such as $p \vee \neg p$, or a theorem of first-order logic such as $a=a$, without needing actually to use it. The modified rule remains classically correct and one can carry out the same derivations as before without change. Systems of natural deduction that proceed this way, for classical and non-classical logics, are discussed in [3]. But, in the classical context, why bother unless one thinks that there is something philosophically wrong about second-level inference without a supposition? The manoeuvre does not correspond to mathematical practice and detours through an idle or artificially employed assumption.

The "let $x$ be..." locution. In practice, when one uses a phrase like 'Let $x$ be an arbitrary so-and-so' (say, an arbitrary equilateral triangle) the 'so-and-so' condition almost always identifies a class that is more restricted than the entire universe of discourse under consideration (say, the class of points, lines and figures on a plane). This is because we are doing two things at the same time. We are trying to prove, from given information $\Gamma$ (say, the axioms of plane geometry), a general conditional $\forall x(\varphi(x) \rightarrow \psi(x))$. To that end, we begin by establishing $\psi(x)$ from $\Gamma \cup\{\varphi(x)\}$ and then carry out two second-level steps. The first applies $C P$ to conclude that $\Gamma \vdash \varphi(x) \rightarrow \psi(x)$, to which the second applies $U G$, under the condition that $x$ does not occur free in $\Gamma$, to conclude that $\Gamma \vdash \forall x(\varphi(x) \rightarrow \psi(x))$. To streamline the argument, the two steps are customarily run together.

Non-classical logics. Although we are concerned only with classical reasoning, we note in passing that supposition-free second-level inference rules also appear in some well-known non-classical logics, notably for introducing the box connective in natural deduction systems for the modal logic $S 5$ and some of its sub-logics, as well as for logics of relevance-sensitive conditionals when those conditionals are understood as conveying some kind of necessity (as is the case for the relevance logic $E$, but not for $R$ ).

For $S 5$, that is not really anything new since its box can be seen as shorthand for a universal quantifier with a single fixed variable $x$. Specifically, modal formulae can be translated to classical first-order formulae with monadic predicates: fix a variable $x$, associate injectively each sentence letter $p$ with a one-place predicate letter $P$, put $\mathcal{T}(p)=P(x)$ for sentence letters (always with the fixed choice of variable $x$ ), translate truth- 
functional connectives into themselves, and put $\mathcal{T}(\square \alpha)=\forall x \mathcal{T}(\alpha)$. When the $S 5$ rule $\square^{+}$is expressed in the form $\Gamma \vdash \gamma / \Gamma \vdash \square \gamma$ under the proviso that no sentence letter in $\gamma$ occurs unmodalized in $\Gamma$, it corresponds to classical $U G$. In this way, $U G$ can be seen as the ultimate source of supposition-free second-level rules in $S 5$ and thus also, indirectly, in some of its subsystems.

\section{Indirectness}

The best-known of the perfumes, used and discussed since Greek antiquity, is indirectness. As it has received so much attention, we will be very brief.

Often, reductio ad absurdum alone is counted as indirect, but it seems reasonable to include contrapositive conditional proof under this name, as is sometimes done. Accordingly, we define a second-level inference form $\Gamma_{i} \vdash \gamma_{i}(i \leq n) / \Delta \vdash \delta$ to be indirect iff each subordinate argument has a supposition that negates (or is negated by) the conclusion (or the consequent of the conclusion) of the principal argument. Indirect inferences are thus by definition a particular type of suppositional inference. Inspection tells us that in this sense $R A A a, R A A b$ and $C P b$ are indirect, while the other argument-forms in Table 1 are direct.

Reductio often permits very short and elegant arguments; see e.g. [7, section 2.3] for a collection of examples. In the present author's view, this is not so much due to the manipulation of negation as to the fact, that it is both suppositional and, at least for the more elegant applications of $R A A a$, goal-simplifying in the sense defined in section 2 and discussed further in the section 4 . On the negative side, reductio proofs for $\forall \exists$ statements are sometimes non-constructive (no witness provided for the existential quantification) which, notoriously, makes them unfriendly to computation; it has also sometimes given rise to philosophical doubts about its legitimacy (see e.g. [12]).

For $C P b$, where the desired conclusion is of the form $\alpha \rightarrow \beta$, the inferential convenience of $\neg \beta$ as a supposition with $\neg \alpha$ as goal in the subordinate inference can be greater, less, or about the same as, that of a corresponding application of $C P a$ where $\alpha$ is supposition and $\beta$ is goal, depending on the internal logical structure of those propositions.

The extent to which mathematicians use indirect inference is partly a matter of personal style. Reticence about $C P b$ is not common, but some 
prefer not to bring in the reductio artillery except when it provides major benefits. On the other hand, others deploy it routinely. A mixed strategy is to give an indirect inference if it is succinct and, if a witness is missing or computation blocked, accompany it by a longer and perhaps more intricate constructive argument, if one can be found.

Of course, direct second-level inference forms can always be rendered indirect. For example, the first trick mentioned in section 2 for making $U G$ suppositional, adding $\neg \gamma$ to the premises of the subordinate inference $\Gamma \vdash \gamma$, at the same time makes it indirect. However, as mentioned there, this manoeuvre does not correspond to mathematical practice, being artificial with an unnecessary detour. One occasionally sees a less generic, but more interesting, indirect variant of $D P$ that eliminates one of the two disjuncts, namely the rule: $D P y: \Gamma \cup\{\alpha\} \vdash \gamma ; \Gamma \cup\{\beta\} \vdash \delta ; \Gamma \cup\{\beta\} \vdash \neg \delta / \Gamma, \alpha \vee \beta \vdash \gamma$. However, from a conceptual point of view, we see this as a combination of standard $D P$ with reductio and, for this reason, do not include it in Table 1.

Why, then, have we given $C P b$ a seat at that table, since it too can be seen as conceptually composite, a combination of $C P a$ with a first-level inference? The reason is practical rather than formal: $C P b$ is extremely common in everyday mathematical reasoning, while the indirect form of disjunctive proof is very much less so. But nothing prevents the reader from extending Table 1 with rows for rules such as $D P y, D P x$ (section 2) or any other second-level inference forms that can reasonably claim to be deployed in inferential practice.

\section{Goal-simplification}

In section 2, we observed in passing that although $U G$ is not suppositional, it is conclusion-simplifying (more briefly, goal-simplifying), in the sense that the conclusion $\gamma$ of the subordinate argument is strictly logically simpler than the conclusion $\forall x(\gamma)$ of the principal argument. Evidently, this property facilitates inference, by reducing the complexity of what we have to prove.

Not many patterns of second-level inference in everyday mathematical reasoning have this property. $D P$ and $E I$ fail it, as the conclusions of their subordinate inferences are the same as the conclusions of their respective principal inferences, thus not strictly simpler. On the other hand, con- 
ditional proof is a big goal-simplifier. This is patently so in the case of $C P a$, where the conclusion $\beta$ of the subordinate inference is only the consequent of the conclusion $\alpha \rightarrow \beta$ of the principal one. It is less clear for the contrapositive version $C P b$, for the negation sign in the conclusion $\neg \alpha$ of the subordinate inference does not appear in the conclusion $\alpha \rightarrow \beta$ of the principal one. However, the elimination of the arrow intuitively compensates for the addition of a negation: we can understand logical complexity in a way that gives arrows more weight than negations (as is sometimes done when defining the notion of a subformula for inductive arguments in proof-theory), thereby also treating $C P b$ as goal-simplifying.

$R A A a$ is not in general a goal-simplifier, but it is so in some instances. The conclusions $\gamma, \neg \gamma$ of the two subordinate arguments may have any complexity at all compared to the conclusion $\alpha$ of the principal inference but, in practice, they are often simpler. As we are concerned with practice as well as form, we put $0 / 1$ in this cell of Table 1 .

Thus, conditional proof in both forms $C P a, C P b$ and $R A A a$ (in some of its applications) are the only second-level inference rules of Table 1 that are both suppositional and goal-simplifying. The additional power brought by the availability of a supposition, combined with the reduced complexity obtained by goal-chipping, iterated as many times as occasion arises, can transform a complex inferential task into a trivial one. That is surely part of the reason why those three rules are such great work-horses, stars of the second-level stable.

\section{Goal-preservation and wlog reasoning}

Call a second-level inference rule $\Gamma_{i} \vdash \gamma_{i}(i \leq n) / \Delta \vdash \delta$ conclusion-preserving (briefly, goal-preserving) iff $\gamma_{i}=\delta$ for all $i \leq n$. By definition, the goalpreserving rules are disjoint from the goal-simplifying ones, which make the conclusion strictly simpler. They are also disjoint from the indirect ones, which radically modify the conclusion. Clearly, $D P, E I$ and $R A A b$ are goal-preserving while $U G, C P a, C P b$ and $R A A a$ are not.

Thus, all of the goal-preserving rules for connectives that we consider in Table 1 turn out to be suppositional. To find a non-suppositional goalpreserving rule, one can turn to the connective-free rule of monotony. Indeed, that is essentially the only manner in which the combination can hold for, quite generally, a second-level rule $\Gamma_{i} \vdash \gamma_{i}(i \leq n) / \Delta \vdash \delta$ that is 
non-suppositional must have some $\Gamma_{j} \subseteq \Delta$ while, if it is goal-preserving, it must put each $\gamma_{i}=\delta$. Thus, it must be of the form $\Gamma_{j} \vdash \delta ; \Gamma_{i} \vdash \delta(j \neq i \leq$ $n) / \Gamma_{j} \cup \Gamma^{\prime} \vdash \delta$, which is just monotony possibly accompanied by additional, but redundant, subordinate inferences.

Goal-preservation underlies a kind of reasoning that is familiar in mathematical practice but seldom discussed in logic textbooks. It is typically introduced by a such as "assume without loss of generality that...", abbreviated as "assume wlog that...". This tells us that we are making a supposition in a (single) subordinate inference, with conclusion unchanged. In general, the assumption is mathematically substantive.

A well-known example arises when one is working with well-ordered sets. In that context, a standard wlog move is to say, when there is an $x$ with a certain property, that we can choose such an $x$ and assume wlog that it is least among them (or minimal among them, in the more general case of well-founded sets).

Although content-specific, this example has an interesting parallel with the logical rule $E I$. It may be understood as a second-level inference pattern that is available when $\Gamma$ tells us, inter alia, that the domain of discourse is well-founded by a relation $<$ :

$E I_{<}: \Gamma, \alpha_{<} \vdash \gamma / \Gamma, \exists x(\alpha) \vdash \gamma$, when $x$ has no free occurrences in $\Gamma, \gamma$.

Here $\alpha_{<}$abbreviates $\alpha \wedge \neg \exists y\left(y<x \wedge \alpha_{x:=y}\right)$, where $\alpha_{x:=y}$ is the result of substituting a variable $y$ not occurring in $\alpha, \gamma$ or $\Gamma$, for all free occurrences of $x$ in $\alpha$. The only difference between this and $E I$ as formulated earlier, is that $\alpha_{<}$replaces $\alpha$ as premise of the subordinate inference. Trivially, $\alpha_{<} \models \alpha$, so $E I_{<}$is more powerful than plain $E I$. It can be regarded as running $E I$ together with modus ponens in the context of a well-founded domain, that is, where $\Gamma \models \exists x(\alpha) \rightarrow \exists x\left(\alpha_{<}\right)$. The merging can render presentation more elegant; in particular, it often permits us to halve the number of different variables that are needed for clear exposition.

While the above example of wlog reasoning is closely related to $E I$, there are many others that appear to be less so. For example, if we want to show that every Boolean algebra has a certain property that we know to be preserved under Boolean isomorphisms, then we may consider an arbitrary Boolean algebra $B$ and assume without loss of generality that it is a field of sets. This is because we have a representation theorem telling us that every Boolean algebra is isomorphic to some field of sets. However, 
care needs to be taken since, notoriously, some interesting properties of Boolean algebras are not preserved under isomorphisms, for example, the property of infinite distributivity (see e.g. [13, section 35]).

In this example, the parallel with EI reappears if the wlog step is made in the course of an indirect proof: supposing that there is a Boolean algebra $B$ that lacks a certain property, we seek a contradiction; if the property is preserved under isomorphism, then we may suppose wlog that B is a field of sets, and continue to contradiction from there.

For examples further away from logic and set theory, see e.g. [2]. For aficionados of non-classical logic, there is an interesting use of a wlog procedure in the construction of relevance-sensitive truth-trees. When the arrow connective is understood as relevance-sensitive, then decomposition of $\neg(\varphi \rightarrow \psi)$ into $\varphi, \neg \psi$ on a branch of a decomposition tree is no longer an act of first-level inference, as it is for classical truth-trees; it is a wlog second-level step. See [8, chapter 11], with a more detailed account in [9].

\section{Premise-preservation}

Evidently, one may define a dual to goal-preservation. Call a second-level inference rules $\Gamma_{i} \vdash \gamma_{i}(i \leq n) / \Delta \vdash \delta$ premise-preserving iff $\Delta \subseteq \Gamma_{i}$ for all $i \leq n$. In [8, chapter 10], this property was called "incrementality".

Inspection of the rules in Table 1 shows that $U G, C T, C P$ (both forms) and $R A A$ (both forms) are premise-preserving. On the other hand, $E I$ and $D P$ are not since, in general, $\exists x(\alpha) \notin \Gamma \cup\{\alpha\}$ and $\alpha \vee \beta \notin \Gamma \cup\{\alpha\}, \Gamma \cup\{\beta\}$.

On the other hand, the rather special form of $D P$ that we called $D P x$ (section 2) is premise-preserving. Moreover, since trivially $\alpha \models \alpha \vee \beta$, $\beta \models \alpha \vee \beta, \alpha \models \exists x(\alpha)$, both $D P$ and $E I$ may both be formulated in equivalent, but redundant, ways that are premise-preserving:

$$
\begin{array}{r}
\Gamma \cup\{\alpha \vee \beta\} \cup\{\alpha\} \vdash \gamma ; \Gamma \cup\{\alpha \vee \beta\} \cup\{\beta\} \vdash \gamma / \Gamma \cup\{\alpha \vee \beta\} \vdash \gamma \\
\Gamma \cup\{\exists x(\alpha)\} \cup\{\alpha\} \vdash \gamma / \Gamma \cup\{\exists x(\alpha)\} \vdash \gamma, \text { when } x \text { has no free } \\
\text { occurrences in } \Gamma, \gamma .
\end{array}
$$

To mark the fact that these variant formulations of $D P, E I$ are premisepreserving while the standard forms are not, we write 0,1 in the corresponding cells of Table 1.

It is perhaps not immediately obvious, as it was with suppositionality and goal simplification, how premise preservation can be of assistance in 
Table 1. Selected perfumes for familiar second-order rules

\begin{tabular}{|c|c|c|c|c|c|c|}
\hline & & $\begin{array}{c}\text { Supposi- } \\
\text { tional }\end{array}$ & $\begin{array}{l}\text { Goal- } \\
\text { simpli- } \\
\text { fying }\end{array}$ & Indirect & $\begin{array}{c}\text { Goal- } \\
\text { preser- } \\
\text { ving }\end{array}$ & $\begin{array}{c}\text { Pre- } \\
\text { mise- } \\
\text { preser- } \\
\text { ving }\end{array}$ \\
\hline$U G$ & & 0 & 1 & & 0 & 1 \\
\hline$D P$ & & & & 0 & 1 & 0,1 \\
\hline EI & & & & & & 0,1 \\
\hline$C P, \rightarrow^{+}$ & $C P a$ & & 1 & & & \\
\hline & $C P b$ & & & & 0 & 0 \\
\hline$B A A$ & $R A A a$ & & $0 / 1$ & 1 & & \\
\hline & $R A A b$ & & 0 & & 1 & \\
\hline Mon & ony & 0 & 0 & 0 & 1 & 0 \\
\hline C & & & & & 0 & 1 \\
\hline
\end{tabular}

The entries $0 / 1$ and 0,1 in certain cells are explained in the corresponding sections. The acronym EI is for "existential instantiation"; the reader should be warned that some textbook presentations of natural deduction use the same acronym for the first-level rule of "existential introduction".

the business of deduction. We suggest that it can be helpful, illustrating with the premise-preserving rule $C P a$. Consider a situation where we are working within a mathematical theory that is axiomatized by a set $\Gamma$ of propositions, from which a considerable number of consequences have been derived by many hands over a long period of time, and that we wish to prove a conditional proposition $\alpha \rightarrow \gamma$. We take $\alpha$ as a supposition and seek to get $\gamma$ from it, making free use of anything that has already been obtained from $\Gamma$. If we succeed, then we can apply $C P a$ (along with implicit appeal to cumulative transitivity and monotony) and we are done. 
But what would the situation be like if instead of $C P a: \Gamma \cup\{\alpha\} \vdash$ $\gamma / \Gamma \vdash \alpha \rightarrow \gamma$ we had a rule $\Gamma^{\prime} \cup\{\alpha\} \vdash \gamma / \Gamma \vdash \alpha \rightarrow \gamma$ that is not-premisepreserving, that is, where $\Gamma \nsubseteq \Gamma^{\prime}$ ? When carrying out the subordinate inference $\Gamma^{\prime} \cup\{\alpha\} \vdash \gamma$, we would not know, without re-checking, which of the many theorems already deduced from $\Gamma$ are also available for use in the sub-proof. In cases where there are elements of $\Gamma$ that are not trivially implied by $\Gamma^{\prime}$, that checking could be arduous indeed.

\section{Conclusion}

Second-level inference rules come with or without various features that we have dubbed 'perfumes': suppositionality, indirectness, goal-simplification, goal-preservation and premise-preservation. The presence of these perfumes tends to confer practical advantages in the articulation and communication of mathematical inference, each perfume with its own advantage. Familiar rules of mathematical practice, as recorded in Table 1, all have at least one of the perfumes, sometimes more. Conditional proof in both its direct and contraposed forms $(C P a, C P b)$ as well as reductio ad absurdum in its standard form $(R A A a)$, are particularly well endowed in this respect, which may explain why they are such great work-horses. Goal-preservation is also an essential part of wlog reasoning in mathematics, with some examples having analogies to $E I$.

Acknowledgements. Thanks to Alex Citkin, Lloyd Humberstone, Andrzej Indrzejczak, Alexei Muravitsky and Xavier Parent for vigorous comments and criticisms on a draft, and an anonymous referee for helpful suggestions. Apologies to Nefzawi and his English translators, as well as to Jorge Luis Borges, for the title.

\section{References}

[1] G. Gentzen, Untersuchungen über das logische Schliessen, Mathematische Zeitschrift, vol. 23 (1934), pp. 176-210, 405-431, DOI: http: //dx.doi.org/10.1007/BF01201353, English translation: Investigation into logical deduction, pp. 68-131 of The Collected Papers of Gerhard Gentzen, ed. M. E. Szabo. North-Holland, Amsterdam, 1969. 
[2] J. Harrison, Without loss of generality, [in:] S. Berghofer, T. Nipkow (eds.), Theorem-Proving in Higher Order Logics, vol. 5674 of Lecture Notes in Computer Science, Springer, Berlin (2009), pp. 43-59, DOI: http://dx. doi.org/10.1007/978-3-642-03359-9_3.

[3] A. Indrzejczak, Natural Deduction, Hybrid Systems and Modal Logic, Springer, Dordrecht (2010), DOI: http://dx.doi.org/10.1007/978-90481-8785-0.

[4] A. Indrzejczak, Natural Deduction, Internet Encyclopedia of Philosophy, (2015), URL: https://iep.utm.edu/nat-ded/, consulted 16.07.2020.

[5] S. Jaśkowski, On the rules of suppositions in formal logic, Studia Logica, vol. 1 (1934), pp. 1-32.

[6] D. Kalish, R. Montague, Logic: Techniques of Formal Reasoning, Harcourt Brace, New York (1964), also second edition with co-author G. Mar, Oxford University Press, 2001.

[7] J. Lucas, Introduction to Abstract Mathematics, 2nd ed., Rowman \& Littlefield, Maryland, USA (1990).

[8] D. Makinson, Sets, Logic and Maths for Computing, 3rd ed., Undergraduate Topics in Computer Science, Springer, London (2020), DOI: http://dx.doi.org/10.1007/978-1-4471-2500-6.

[9] D. Makinson, Relevance-sensitive truth-trees, [in:] Alasdair Urquhart on Nonclassical and Algebraic Logic and Complexity of Proofs, Outstanding Contributions to Logic, Springer, Berlin (2021), to appear.

[10] J. Pelletier, A brief history of natural deduction, History and Philosophy of Logic, vol. 20 (1999), pp. 1-31, DOI: http://dx.doi.org/10.1080/ 014453499298165.

[11] J. Pelletier, A. P. Hazen, A history of natural deduction, [in:] D. Gabbay, F. J. Pelletier, E. Woods (eds.), Handbook of the History of Logic, vol. 11, North-Holland, Amsterdam (2012), pp. 341-414, DOI: http://dx. doi.org/10.1016/B978-0-444-52937-4.50007-1.

[12] E. Schechter, Constructivism is difficult, American Mathematical Monthly, vol. 108 (2001), pp. 50-54, DOI: http://dx.doi.org/10.1080/ 00029890.2001.11919720.

[13] R. Sikorski, Boolean Algebras, 2nd ed., Springer, Berlin (1964), DOI: http://dx.doi.org/10.1007/978-3-662-01507-0. 
David Makinson

Les Etangs, B2

Domaine de la Ronce

92410 Ville d'Avray, France

e-mail: david.makinson@gmail.com 